\title{
Dinámica de red social y alteración psicológica en adolescentes con ausencia de familia de origen
}

\section{Social network dynamics and psychological alteration in ado- lescents without family of origin}

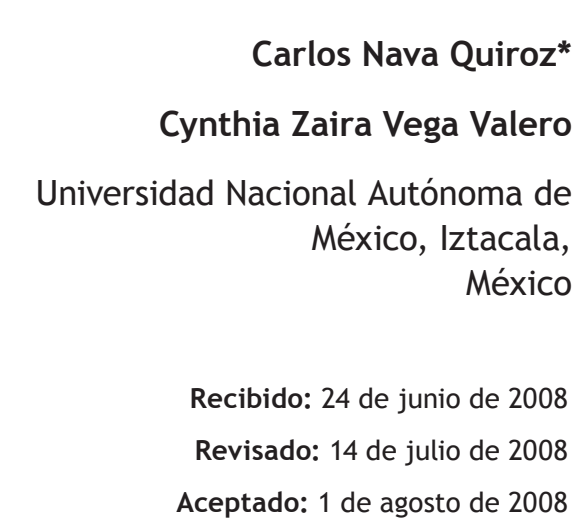

* Correspondencia: Carlos Nava Quiroz, Bosques de Vincenes 4, casa 15, Bosques de Lago, Cuautitlán Izcalli, Edo. de Méx. CP 54760. Correo electrónico: canaqi@servidor.unam.mx. Cynthia Zaira Vega Valero: czaira.vega@correo.unam.mx

\section{Resumen}

El objetivo fue realizar un estudio en muestras de adolescentes que vivieran con sus familias y que no vivieran con ellas, en el cual se analizarán las variables de red y de alteración psicológica (depresión) y probar el valor relativo de las propiedades dinámicas de la red para describir y predecir alteración emocional. Participaron 61 adolescentes, 31 del reclusorio para menores infractores y 30 estudiantes. Los adolescentes recluidos muestran más depresión que los estudiantes y estos últimos obtuvieron puntajes más altos en el índice de relaciones familiares y en la calidad de red. Esto indica que cuando algún componente de la red social se ve alterado, hay más propensión a que se presenten problemas de alteración psicológica, como la depresión, lo que tiene impacto en las medidas de red social.

Palabras clave: red social, calidad de red, depresión, adolescentes, familia de origen. 


\section{Abstract}

This work was to conduct a study with samples of adolescents. One sample was integrated by adolescents who lived with their families while in a second sample they didn't live with them. With regard to the social network and psychological alteration variables (depression) and to prove the relative value of the dynamic properties of the social network to describe and to predict emotional alteration. 61 adolescents participated, 31 were from the prison Readaptation and 30 were students. The confined adolescents showed more depression than student, and obtained higher scores in the Index of Family Relationships and in the Quality social network. The above-mentioned results suggest that when some component of the social network is altered, this fact generates problems of psychological alteration, such depression, and this has an impact in the measures of the social network.

Key words: social network, quiality network, depression, adolescents, origin family.

\section{Introducción}

Es común que al funcionamiento familiar se le atribuya responsabilidad en el desarrollo del individuo. Diferentes estudios empíricos muestran una estrecha relación entre el funcionamiento familiar positivo y el ajuste social y psicológico (Valenzuela, Hernández-Guzman \& Sánchez-Sosa, 1995). Sin embargo, no sólo el funcionamiento familiar se relaciona con el ajuste social y psicológico, las relaciones extra familiares y variables atributivas (edad, sexo, estatus económico, etc.), sino que también otras lo hacen (Bussing et al., 2003).

En particular, la red social, y su tamaño, está asociada con diferentes padecimientos psiquiátricos y la severidad de éstos (Pattison, 1977) o con desajuste social (Shinn, KnicKman \& Weitzman, 1991); otros estudios de carácter epidemiológico prospectivo, a gran escala (Berkman \& Syme, 1979; House, Landis \& Umberson, 1988) reportan que el tamaño de red predice mayor riesgo de muerte en sujetos con pocos vínculos sociales. De manera consistente, los estudios epidemiológicos muestran una relación entre tamaño de red y mortalidad, por ejemplo, en los estudios de Blazer (1982), House, J.S., Landis, K.R y Umberson, D. (1988), Orth-Gomer y Johnson (1987) la mortalidad es alta entre aquéllos que presentan aislamiento social, por lo tanto, con el aumento de los vínculos sociales el riesgo de muerte disminuye. Lo que sugiere que los individuos que poseen una red de relaciones muy cohesionada y que pro- porciona ayuda, tienden a obtener mayores beneficios tanto en la salud como en lo emocional (Cobb, 1976; Sarason, Sarason \& Pierce, 1990).

En otros trabajos (Nava, Vega \& Guerrero, 2002; Nava \& Vega, 2006) se reporta que a pesar de que los individuos neuróticos y alcohólicos están en tratamiento y con su padecimiento controlado, siguen teniendo redes sociales más pequeñas que sus contrapartes no neuróticos o no alcohólicos, aún más, las redes de los individuos sin problemas (Nava, Reyes \& Vega, 2001) que presentaron como condición ser padres de familia jóvenes -y que tenían hijos pequeños- no difieren, de manera sustancial, de las redes de los alcohólicos y neuróticos: lo que conduce a pensar que el tamaño de red es una condición necesaria, pero no suficiente para la cabal comprensión de la red social.

No sólo el tamaño de la red social es útil como predictor, sino también las propiedades dinámicas de la red tienen una función importante, esto es, la calidad de las relaciones sociales en la red (en la modalidad de apoyo social y calidad de red) muestran que sentirse amados y la ayuda instrumental y emocional están relacionadas con la longevidad y con satisfacción de vida, más que el tamaño de red (Berkman, Leo-Summers \& Horwitz, 1992; Seeman \& Syme, 1987), mientras que el aislamiento social y la depresión están asociados con niveles bajos de ayuda instrumental y emocional, así como con pocas relaciones estrechas con otros sujetos (Chou, 1999; Lee \& Crittenden, 1996; Oxman \& Hull, 1997; Vandervoort, 1999). 
Por otra parte, hay estudios que muestran la importancia de la red social familiar en el desarrollo de conductas disruptivas (Wolchik, Ruehlman, Braver \& Sandler, 1989; Wolchik, Sandler \& Braver, 1987); la familia y las habilidades conductuales de supervivencia (Blazer, 1982; Ingersoll \& Antonucci, 1988) y la familia como primer fuente de apoyo social en diferentes niveles (Millar \& Darlington, 2002; Takeda et al, 2004). Otros afirman que cuando los individuos pierden a la familia -por ejemplo a causa de guerras o desastres naturales- presentan episodios depresivos muy severos (Chen, Chung, Chen, Fang \& Chen, 2003; Suar, Damodar, Mandal \& Khuntia, 2002) o cuando la familia se encuentra lejos por razones de trabajo (Salgado \& Maldonado, 1993). También existen reportes que muestran que personas ajenas a la familia son importantes como parte de la red (Peretti, 1980; Salgado \& Maldonado, 1993).

El examen de las redes sociales incluye la evaluación de las propiedades estructurales, tales como tamaño y densidad, así como los aspectos cualitativos, descritos en términos de la calidad de las relaciones con los miembros de la red, lo que incluye el ambiente familiar y la calidad de la red con otros miembros. Estudios previos aseveran que la calidad de la red, en su modalidad de apoyo social se asocia, de manera sistemática, con depresión en adultos (Antonucci, Fuhrer \& Dartigues, 1977; Oxman, Berkman, Kasl, Freeman \& Barrett, 1992) y adolescentes (Chou, 1999), pero no siempre con las variables estructurales de la red; en esencia, son estudios que trabajan con adultos y jóvenes con pertenencia familiar explícita.

En resumen, en la investigación de la red social, su tamaño es el indicador de ajuste psicológico o social por excelencia, en conjunto la calidad y el funcionamiento familiar son los elementos centrales para llegar a una mayor comprensión de la dinámica de la red. Bajo estas circunstancias, la red familiar es el elemento más próximo y que más ayuda al individuo (ver, por ejemplo, los estudios de Pattison, 1977) en periodos de crisis, seguido de otros sujetos significativos que no pertenecen a la familia y que también son importantes para el funcionamiento psicológico o social del individuo. Por lo anterior, cuando las personas poseen altos índices de red social, calidad de red y funcionamiento familiar tienden a presentar mejor ajuste psicológico que aquéllos que muestran deficiencias en alguno de los componentes de la red social, por ejemplo, el caso de sujetos que aunque tienen familia viven alejados de ella por alguna circunstancia.

Por lo anterior, el objetivo del presente proyecto fue realizar un estudio en muestras contrastadas, de individuos que vivieran con su familia e individuos que no vivieran con su familia, con respecto a las variables de red y de alteración psicológica (depresión) y probar el valor relativo de las propiedades dinámicas de la red para describir y para predecir alteración emocional. Se espera que existan diferencias entre los niveles de depresión entre las muestras, así como en los puntajes de red y de ambiente familiar.

\section{Método}

\section{Participantes}

Se realizó el estudio con 61 adolescentes divididos en dos grupos; el primer grupo, 31 adolescentes recluidos en el centro de readaptación para menores infractores de la ciudad de México (promedio de reclusión en meses 13,71 y D.S. 7,34), con edad promedio de 17,58 años; segundo grupo, estudiantes del Colegio de Ciencias y Humanidades plantel Naucalpan (CCH) que fueron homogeneizados de acuerdo con el primer grupo en las variables de edad $(17,77)$, sexo (el $100 \%$ fueron hombres) y grado de estudios ${ }^{1}$.

\section{Diseño}

Dos grupos independientes con muestras no aleatorias intencionales.

\section{Instrumentos}

Inventario de Redes de Interacción Social, que evalúa tamaño de red y sub agrupaciones, con niveles de fiabilidad de test retest de $\mathbf{8 6}$. Índice de

1 En este caso se consideró que el nivel de carrera técnica que cursaban los adolescentes recluidos era equiparable al nivel de bachillerato. 
Relaciones Familiares (FRI) de Moos con alfa de Cronbach de .8068 , en este caso se eliminaron los ítems 6,15 y 12 para la subescala de conflicto; 13 y 19 de la subescala de cohesión; y 14, 17 y 23 de la subescala de expresividad, por sus niveles bajos durante el análisis de fiabilidad, quedando 19 ítems en total. Escala de Calidad de Red (ECAR) con alfa de Cronbach de .8985 , que evalúa calidad de red de familiares, amigos y vecinos. Inventario de Depresión de Beck (fiabilizado y validado, por Conde y Useros, 1975) con alfa de Cronbach de .8621 para este estudio.

\section{Procedimiento}

Los inventarios fueron aplicados en el centro de readaptación social correspondiente, en una sala de 10 por 12 metros; y en el caso de los estudian- tes en el salón de clase, en ambos casos se les pidió que leyeran con cuidado las instrucciones y en caso de tener cualquier duda podían preguntar al instructor; se aclararon las dudas correspondientes con respecto a los cuestionarios.

\section{Análisis estadístico}

Análisis de varianza de una vía, chi cuadrada, descriptivos, correlaciones $r$ de Pearson y análisis discriminante.

\section{Resultados}

El análisis inicial constó de los descriptivos, promedios y desviaciones estándar, para cada una de las escalas con respecto a los sujetos recluidos y externos.

Tabla 1. Descriptivos de las escalas de red, de ambiente familiar y de Beck: jóvenes recluidos y externos del $\mathrm{CCH}$

\begin{tabular}{cccc}
\hline & Adolescentes & Media & D.S. \\
\hline \multirow{2}{*}{ Escala de Beck } & Recluidos & 38,4000 & 10,8615 \\
& $\mathrm{CCH}$ & 28,5333 & 6,1011 \\
Total de red & Recluidos & 22,3226 & 13,4546 \\
& $\mathrm{CCH}$ & 32,1333 & 28,1287 \\
\hline \multirow{2}{*}{ Total de subgrupos } & Recluidos & 20,1290 & 15,8466 \\
& $\mathrm{CCH}$ & 30,8667 & 12,3057 \\
\hline Total de ECAR & Recluidos & 151,3548 & 21,1842 \\
& $\mathrm{CCH}$ & 165,5000 & 18,3505 \\
\hline \multirow{2}{*}{ Calidad de red familiar } & Recluidos & 70,1935 & 9,5409 \\
& $\mathrm{CCH}$ & 75,3667 & 8,9538 \\
\hline \multirow{2}{*}{ Calidad de red amigos } & Recluidos & 56,4839 & 10,1682 \\
\multirow{2}{*}{ Calidad de red vecinos } & $\mathrm{CCH}$ & 65,6667 & 12,1296 \\
\hline \multirow{2}{*}{ Total de FRI de Moos } & Recluidos & 24,6774 & 5,0159 \\
& $\mathrm{CCH}$ & 24,4667 & 4,5541 \\
\hline \multirow{2}{*}{ Total de cohesión } & Recluidos & 33,2333 & 4,0059 \\
& $\mathrm{CCH}$ & 34,0667 & 3,1287 \\
\hline \multirow{2}{*}{ Total de expresividad } & Recluidos & 12,6667 & 1,4933 \\
& $\mathrm{CCH}$ & 12,6000 & 1,8495 \\
\hline Total de conflicto & Recluidos & 9,8333 & 1,8399 \\
& $\mathrm{CCH}$ & 10,2000 & 1,2429 \\
\hline
\end{tabular}

Nota: se presentan en negritas los promedios que fueron mayores para cada variable. 
La tabla 1 muestra que los adolescentes recluidos puntuaron más alto que los de $\mathrm{CCH}$ para la escala de depresión. Mientras que el índice de relaciones familiares y la escala de calidad de red exhiben puntajes más altos para los individuos de $\mathrm{CCH}$ que para los reclusos, con excepción de la subescala de vecinos. En el caso del índice de relaciones familiares, las diferencias fueron mínimas entre ambas muestras. A fin de establecer con mayor certeza si las diferencias observadas eran importantes aplicamos un Análisis de Varianza de una Vía, para todas las variables (ver tabla 2).

Como se puede observar, existen diferencias significativas con respecto a la escala de depresión de Beck, subgrupos de red, calidad de red en general, calidad de red familiar y de amigos, pero no con respecto al total de red, calidad de red de vecinos y las de ambiente familiar (FRI de Moos).

Tabla 2. ANOVA de reclusos vs estudiantes del $\mathrm{CCH}$

\begin{tabular}{lcc}
\hline & $\mathrm{F}_{(1,59)}$ & Sig. \\
\hline Escala de Beck & 18,818 &, 000 \\
\hline Total de red & 3,051 &, 086 \\
\hline Total de subgrupos & 8,697 & .005 \\
\hline Total de ECAR & 7,748 & .007 \\
\hline Calidad de red familiar & 4,761 &, 033 \\
Calidad de red amigos & 10,294 &, 002 \\
\hline Calidad de red vecinos &, 029 &, 864 \\
\hline Total de FRI de Moos &, 806 &, 373 \\
\hline Total de cohesión &, 024 &, 878 \\
\hline Total de expresividad &, 818 &, 369 \\
\hline Total de conflicto & 2.481 &, 121 \\
\hline
\end{tabular}

Ya que las variables de ambiente familiar no fueron significativas, como información adicional, se evaluó si existían diferencias entre las variables demográficas de con quién vivían los sujetos. Para tal efecto se aplicaron pruebas chi cuadrada para contrastar uno a uno los integrantes de la red con quienes reportaban vivir los sujetos recluidos antes de ingresar al penal y con quién vivían los estudiantes de $\mathrm{CCH}$, los análisis mostraron diferencias significativas para el caso del padre $\left(X^{2}=\right.$ $7,425, p=, 006)$ y madre $\left(X^{2}=7,346, p=, 007\right)$ lo que indica que los sujetos recluidos vivían ya fuera con el papá o con la mamá, en muchos casos, pero no con ambos; para el resto de los contrastes (hermanos, tíos, abuelos, amigos, solo) no existieron diferencias. También se analizó la relación entre la cantidad de personas que visitaba a los sujetos recluidos y las variables de red y depresión; no existieron correlaciones significativas al respecto.

Por otra parte, se correlacionaron los puntajes de depresión y calidad de red, de lo que resultaron significativas las correlaciones entre la subescala de calidad de red familiar y escala de Beck $(r=-, 559 ; p<0,001)$, escala de calidad de red de amigos y escala de calidad de red de familiar ( $r=$ ,603; $p<0,001$ ) para los sujetos recluidos, mientras que para los estudiantes de bachillerato la escala de calidad de red familiar correlacionó con la de Beck $(r=-, 454, p<0,05)$ y con los subgrupos de red $(r=, 506 ; p<0,001)$. Las correlaciones fueron muy bajas y no significativas entre el tiempo de reclusión y las variables que resultaron significativas en el ANOVA.

Para concluir, se tenía la inquietud de precisar qué tan pertinentes eran las variables de red y depresión para predecir la pertenencia de los sujetos a los grupos muestra, por lo cual se realizó un análisis discriminante primero con las variables de red y después incluyendo la escala de Beck (ver tablas 3 y 4 ).

Las variables que resultaron significativas -calidad de red- en el análisis de varianza (tabla 2) fueron incluidas en el análisis discriminante y a partir de ellas fue factible asignar a su grupo de pertenencia al $77 \%$ de los participantes. Cuando, además de las variables de calidad se incluyó a la de depresión, sólo hubo una diferencia del $1,3 \%$, en comparación con la inclusión de todas las escalas más la de depresión. 
Tabla 3. Clasificación de resultados del análisis discriminante para predicción de pertenencia al grupo de acuerdo con las variables de red

\begin{tabular}{lccc}
\hline \multicolumn{1}{c}{ Grupo } & $\begin{array}{c}\text { Predicción de } \\
\text { pertenencia al grupo }\end{array}$ & $\begin{array}{c}\text { Predicción de } \\
\text { pertenencia al grupo }\end{array}$ & Total \\
\hline Conteo de adolescentes recluidos & Adolescentes recluidos & Adolescentes CCH & \\
\hline Conteo de adolescentes del CCH & 24 & 7 & 30 \\
Porcentaje de adolescentes recluidos & 7 & 23 & 30 \\
Porcentaje de adolescentes $\mathrm{CCH}$ & 77,4 & 22,6 & 100,0 \\
\hline
\end{tabular}

Nota: $77 \%$ de los casos agrupados originalmente fueron correctamente clasificados.

Tabla 4. Clasificación de resultados del análisis discriminante para predicción de pertenencia al grupo de acuerdo con las variables de red y Beck

\begin{tabular}{lccc}
\hline \multicolumn{1}{c}{ Grupo } & $\begin{array}{c}\text { Predicción de pertenencia } \\
\text { al grupo }\end{array}$ & $\begin{array}{c}\text { Predicción de } \\
\text { pertenencia al grupo }\end{array}$ & Total \\
\hline \multicolumn{1}{c}{ Adolescentes recluidos } & Adolescentes CCH & \\
\hline Conteo de adolescentes recluidos & 22 & 8 & 30 \\
Conteo de adolescentes del CCH & 5 & 25 & 30 \\
$\begin{array}{l}\text { Porcentaje de adolescentes } \\
\text { recluidos }\end{array}$ & 73,3 & 26,7 & 100,0 \\
Porcentaje de adolescentes CCH & 16,7 & 83,3 & 100,0 \\
\hline
\end{tabular}

Nota: $78,3 \%$ de los casos agrupados originalmente fueron correctamente clasificados.

\section{Discusión}

El propósito de este estudio fue mostrar que cuando algún componente de la red social se altera tienden a presentarse problemas de alteración psicológica (como depresión) y en las medidas de red social. En este caso, se eligieron muestras de sujetos que contrastan en algún aspecto trascendente de la red, para el caso la red familiar. Se cuidó de homogeneizar aquellas variables que pudieran afectar los resultados, tales como edad, sexo, nivel socioeconómico, etc. Las razones que influyeron para que se eligiera una muestra de sujetos recluidos fue que los jóvenes eran obligados, por sus circunstancias delictivas, a permanecer lejos de la familia; este alejamiento involuntario inter- fiere con el contacto interpersonal cotidiano que es necesario para construir una relación óptima de red, por lo menos, familiar. Por lo anterior se puede afirmar que, en general, los resultados de esta investigación concuerdan con estudios previos acerca de la alteración psicológica (depresión) y niveles bajos en la calidad de red social (Chou, 1999; Lee \& Crittenden, 1996).

En particular, tomar dos muestras que difirieran con respecto a uno de los componentes de la red social (estar lejos de la familia) permitió probar que cuando los individuos son privados del contacto regular con su red, concretamente la familiar, tienden a presentar alteraciones psicológicas (depresión), este hecho concuerda con otros estudios 
(Chou, 1999, Lee \& Crittenden, 1996) en el sentido de encontrar niveles altos de depresión en sujetos aislados, aun cuando en este caso el aislamiento es forzado y en los otros es debido a enfermedad u otra circunstancias, como ser inmigrante.

Otro aspecto importante es que se encuentran diferencias específicas en los puntajes de la escala de calidad de red, en especial en las sub escalas de calidad de red familiar y calidad de red con amigos. Distintos estudios reportan diferencias en el apoyo social, como medida de calidad de red (Chou, 1999; Antonucci, Fuhrer \& Dartigues, 1977; Barrett, 1992; Oxman et al., 1977) en sujetos que son identificados como depresivos. Aun cuando las medidas de apoyo social y calidad de red difieren en cuanto a los propósitos para lo que fueron creadas, ambas son buenos indicadores de calidad de red. La ventaja de la escala de calidad de red es que fue diseñada ex profeso para evaluar la red social, en este sentido representa una ventaja sobre las escalas de apoyo social, sobre todo, de aquéllas que son muy genéricas. La escala de calidad de red proporciona una visión general de la calidad de la red y, asimismo. permite particularizar sobre componentes específicos de la red (familia, amigos, vecinos).

Con respecto al ambiente familiar, no se encontraron diferencias importantes en este caso, a pesar de que dicho instrumento fue cuidadosamente evaluado, lo cual permite concluir que las variables de clima familiar no difieren o se ven alteradas a pesar del aislamiento de los individuos, lo que pudiera indicar que los sujetos perciben al clima familiar más rígido, como normas de comportamiento más invariantes que caracterizan a los miembros de la familia, mientras que la calidad de red es percibida como maneras de comportamiento más orientadas a la convivencia y ayuda interpersonal.

Otro de los aspectos para destacar es que de las medidas estructurales de red, sólo en los subgrupos, se encontraron diferencias significativas entre las muestras de sujetos, lo que indica mayor diversificación de relaciones (con diferentes grupos) dentro de la red para los estudiantes del $\mathrm{CCH}$; por el contrario, los internos mostraron menos diversificación de relaciones con otros individuos y entre los miembros de su red. Esto se puede deber al mismo estado de reclusión en que se encuentran. En el caso del tamaño de red, a pesar de la diferencia que a simple vista se muestra entre los grupos (casi un tercio de diferencia) no se encuentran diferencias significativas; esto parece contrastar con estudios previos (por ejemplo, Sarason, Sarason \& Pierce 1990; Pattison, 1977), sin embargo, hay que recordar que los tamaños de redes han mostrado ser buenos para predecir padecimientos psicológicos o psiquiátricos severos. Éste no es el caso de los reclusos, es decir, no se podría afirmar que estos sujetos presentan alteraciones emocionales extremas, una razón más es que a pesar de la distancia en el tamaño de red, en ambos casos, los valores caen dentro de las estimaciones que para poblaciones de sujetos normales se han establecido previamente (Nava, 1991; Pattison, 1977; Pattison, Defrancisco, Wood, Frazier \& Crowder, 1975).

En lo referente a las correlaciones, los coeficientes fueron moderados, pero significativos lo que indica que a mayor calidad de red familiar y amigos menor era la depresión en los sujetos recluidos y para los estudiantes ocurrió algo similar, pero con valores inferiores de correlación, lo que da un indicador, aunque sea moderado, de la importancia de la red familiar y de amigos en situaciones de aislamiento.

En el caso de la significación de las variables demográficas, se encontró que sólo existieron diferencias importantes en vivir con los padres entre ambas muestras, lo que está indicando que los componentes estructurales y de calidad de red tienen mayor peso en la comprensión de las relaciones de los individuos con su entorno social que las variables demográficas. A la luz de estos resultados, las variables demográficas parecen predecir de manera colateral el conflicto familiar, pero no son factores explicativos y menos aún dan pie para promover tratamientos de intervención efectivos.

Además, se propuso saber qué tan buenos eran los instrumentos de redes y depresión para predecir la pertenencia de los sujetos a los grupos, para lo cual se realizó un análisis discriminante, 
primero con las variables de red que resultaron significativas y en seguida en conjunto con la escala de depresión. Los resultados mostraron que cuando se agregó la escala de Beck al análisis fue poca la ganancia en el porcentaje de asignación de los sujetos a los grupos, lo que indica la fuerza de discriminación de las variables de red. Este análisis permite tener un grado razonable de confianza en la identificación de sujetos con problemas familiares y de alteración psicológica para poder asignarlos a tratamientos estructurados. Por otra parte, proporciona una prueba adicional de la importancia de las variables de red para identificar alteraciones en la red, las cuales, a su vez, pueden servir como directrices, en el ámbito clínico, para la intervención.

\section{Referencias}

Antonucci, T.C., Furer, R. \& Dartigues, J. (1977). Social Relations and Depressive Symptomatology in a Sample of community-Dwelling French Older Adults. Psychology Aging, 12, 189-195.

Berkman, L.F. \& Syme, S.L. (1979). Social Networks, Host Resistance and Mortality: A Nine Year Follow-Up Study of Alameda County Residents. American Journal of Epidemiology, 109, 186-204.

Berkman, L.F., Leo-Summers, L. \& Horwitz, R.I. (1992). Emotional Support and Survival after Myocar Dial Infarction: A Prospective, Population-Based Study of the Elderly. Annals of Internal Medicine, 117, 1002-1009.

Blazer, D.G. (1982). Social Support and Mortality in an Elderly Community Population. American Journal of Epidemiology, 115 (5), 684-694.

Bussing, R., Zima, B.T., Gary, F.A., Mason, D.M., Leon, C.E., Sinha, K. \& Garvan, W.C. (July $7^{\text {th }}$ 2003). Social Networks, Caregiver Strain and Utilization of Mental Health Service Among Elementary School Students at High Risk For ADHD. Journal of American Academy of Child and Adolescent Psychiatry, 42.
Chen, H., Chung, H., Chen, T., Fang, L., \& Chen, J-P. (April 2003). The Emotional Distress in a Community after the Terrorist Attack on the World Trade Center. Community Mental Health Journal, 39 (2), 157-165.

Chou, K.L. (1999). Social Support and Subjetive Well-Being among Hong Kong Chinese Young Adults. Journal of Genetic Psychology, 160 (3), 319-331.

Cobb, S. (1976). Social Support as a Moderator of Life Estress. Psychosomatic Medicine, 38, 300-314.

Conde, V. \& Useros, E. (1975). Adaptación castellana de la escala de evaluación conductual para la depresión de Beck (y II). Revista de Psiquiatría y Psicología Médica, XII (4), 217-236.

House, J.S., Landis, K.R. \& Umberson, D. (1988). Social Relationship and Health. Science, 242, 540-545.

Ingersoll, B. \& Antonucci, T.C. (1988). Reciprocal and nonreciprocal social support: Contrasting Sides of Intimate Relationships. Journal of Gerontology, 43 (3), 65-73.

Lee, M.S. \& Crittenden, K.S. (1996). Social Support and Depression among Korean Immigrants in the United States. International Journal of Aging \& Human Development, 42 (4), 15-25.

Miller, R.F. \& Darlington, Y. (2002). Who Supports? The Providers of Social Support to Dual-Parent Families Caring for Young Children. Journal of Community Psychology, 30 (5), 461-473.

Nava, Q.C., Reyes, L.I. \& Vega, V.Z. (2001). Estructura y calidad de red social en tres muestras familiares; adolescentes, adultos y jóvenes. Revista de Psicología Social y Personalidad, XVII (1), 17-34.

Nava, Q.C., Vega, V.Z. \& Guerrero, B.J. (2002). Calidad de red y ambiente familiar de neuróticos. En Segundo encuentro latinoamericano de psicología ambiental: Sustentabilidad, 
comportamiento ambiental y calidad de vida. A.A. Terán y O.A Landázuri (compliladoras). UNAM. México.

Nava, Q.C. \& Vega, V.Z. (Diciembre de 2006). Estructura y calidad de red de Alcohólicos Anónimos. Anales de Psicología, 22 (2), 212-216.

Orth-Gomer, K. \& Johnson, J.V. (1987). Social Network Interaction and Mortality: Asix Year Follow-Up Study of a Random Sample of the Swedish Population. Journal of Chronical Diseases, 40, 949-957.

Oxman, T.E. Berkman, L.F., Kasl, S. Freeman, D.H. \& Barrett, J. (1992). Social Support and Depressive Symptoms in the Erderly. American Journal of Epidemiology, 135, 356-368.

Oxman, T.E. \& Hull, J.G. (1997). Social Support, Depression, and Activitis of Daily Living in older Heart Surgery Patients. Journal of Gerontology, 52 (1), 1-14.

Pattison, E.M. (1977). A Theorical-Empirical Base for Social Therapy. En E.F. Fulks, A.R. Westermeyer \& R.M. Wintrob (Eds.). Current perspectives in cultural psychiatry. New York: Spectrum Publ.

Pattison, E.M., Defrancisco, D., Wood, P., Frazier, H. \& Crowder, J. (1975). A Psychosocial Kinship Model for Family Therapy. American Journal of Psychiatry, 132 (12), 1246-1251.

Peretti, P.O. (1980). Perceived Primary Group Criteria in the Relational Network of Closet Friendships- Adolescence, 15, (59), 553-565.

Salgado, N. \& Maldonado, M. (1993). Funcionamiento psicosocial en esposas de emigrantes mexicanos a los Estados Unidos. Revista Latinoamericana de Psicología, 25 (2), 167-180.

Sarason, B.R., Sarason, I.G. \& Pierce, G.R. (1990). Social Support: An Interactional View. New York. Wiley and Sons.
Seeman, T.E. \& Syme, S.L. (1987). Social Network and Coronary Artery Disease: A Comparison of the Structure and Function of Social Relations as Predictors of Disease. Psychosomatic Medicine, 49, 341-354.

Shinn, M., KnicKman J. \& Weitzman, B. (1991). Social Relationhips and Vulnerability to Becoming Homeless among Poor Families. American Psychologist, 46 (11), 1180-1187.

Suar, Damodar, Mandal, M.K. \& Khuntia, R. (October 2002). Supercyclone in Orissa: An Assessment of Psychological Status of Survivors. Journal of Traumatic Stress, 15 (4), 313-319.

Takeda, Y, Kawachia I, Yamagatab Z., Hashimotoc S., Matsumurad Y., Ogurie S. \& Okayama A. (2004). Multigenerational Family Structure in Japanese Society: Impacts on Stress and Health Behaviors Among Women and Men. Social Science and Medicine, 59, 69-81.

Valenzuela, V.J., Hernández-Guzmán, L. \& SánchezSosa, J.J. (1995). Quejas psicosomáticas y comportamiento sexual de adolescentes en función del abuso sexual sufrido en la niñez. Archivos Hispanoamericanos de Sexología, 1 (1), 201-204.

Vandervoort, D. (1999). Quality of Social Support in Mental and Physical Health. Current Psychology, 18 (2), 205-222.

Wolchik, S.A., Ruehlman, L.S., Braver, S.L. \& Sandler, I.N. (1989). Social Support of Children of Divorce: Direct and Stress Buffering Effects. American Journal of Community Psychology, 17 (4), 485-501.

Wolchik, S.A., Sandler, I.N. \& Braver, S.L. (1987). Social Support: Its Assessment and Relation to Children'S Adjustment. In Eisenberg (Ed.). Contemporary Topics in Developmental Psychology. New York: Wiley. 